\title{
DENSITY OF MEMBERS WITH EXTRA HODGE CYCLES IN A FAMILY OF HODGE STRUCTURES*
}

\author{
CHING-LI CHAI ${ }^{\dagger}$
}

\begin{abstract}
Let $M_{K}(G, X)$ be a Shimura variety over $\mathbb{C}$; let $L$ be a $\mathbb{Q}$-subgroup of $G$ whose $G(\mathbb{Q})$-conjugacy class corresponds to a prescribed type of Hodge cycles. For every subvariety $V$ of $M_{K}(G, X)$, denote by $S(V, L)$ the subset of points of $V$ whose Mumford-Tate group is contained in a $G(\mathbb{Q})$-conjugate of $L$. We define an invariant $c(G, X, L)$ depending only on the $G(\mathbb{R})$-conjugacy of $L$. The main result says that for every subvariety $V$ of codimension at most $c(G, X, L)$, the subset $S(V, L)$ is dense in $V$ in the metric topology. The value of $c(G, X, L)$ is tabulated in many examples.
\end{abstract}

0. Introduction. The question we address in this paper is part of a more general question: Given a variation of Hodge $\mathbb{Q}$-structures over a complex analytic variety $V$, do the points of $V$ corresponding to members in this family having extra Hodge cycles of a given type form a dense subset of $V$ ?

A special case of this question was raised in [1]. Let $V \subset \mathcal{A}_{g}$ be a subvariety of the moduli space $\mathcal{A}_{g}$ of principally polarized abelian varieties of dimension $g$ over $\mathbb{C}$, and for any integer $k$ between 1 and $g-1$ denote by $S_{k}(V)$ the subset of $V$ consisting of all points $x \in V$ such that the corresponding abelian variety $A_{x}$ has an abelian subvariety of dimension $k$. In [1], Colombo and Pirola gave a sufficient condition for $S_{k}(V)$ to be dense in $V$ with respect to the metric topology. They also proved that if $V$ is a subvariety of the Jacobian locus $T\left(\mathcal{M}_{g}\right)$ of codimension at most $g-1$, then $S_{1}(V)$ is dense in $V$. Here $T\left(\mathcal{M}_{g}\right)$ denotes the locus of jacobians of complete smooth curves of genus $g$ in $\mathcal{A}_{g}$. Using the same criterion, Izadi showed in [5] that if $V$ is either a subvariety of $\mathcal{A}_{g}$ of dimension at most $g$, or $V$ is a subvariety of $T\left(\mathcal{M}_{g}\right)$ of dimension at most $g$, then $S_{1}(V)$ is dense in $V$.

This paper was started by the attempt to understand the meaning of the results of $[1,5]$ in a more general context. We replace the parameter space $\mathcal{A}_{g}$ by a Shimura variety $M_{K}(G, X)$, and allow Hodge cycles more general than idempotent endomorphisms of a polarized abelian variety to arrive at the more general question stated at the beginning; see $\S 1$ for the precise formulation. In our setting, the subset $S_{k}(V)$ of $V \subset \mathcal{A}_{g}$ is replaced by a subset $S(V, L)$ of $V \subset M_{K}(G, X)$, where $L$ is a reductive subgroup of $G$ defined over $\mathbb{Q}$. We define an invariant $c(G, X, L) \in \mathbb{N}$ which has the property that $S(V, L)$ is dense in $V$ if $V$ has codimension at most $c(G, X, L)$ in $M_{K}(G, X)$. This generalizes $[1,5]$. The invariant $c(G, X, L)$ depends only on the $G(\mathbb{R})$-conjugacy class of $L$ in $G$; its value in the case considered in $[1,5]$ is $g$.

Our method differs from those used in $[1,5]$ only at the end, where we further linearized the problem to compute $c(G, X, L)$. This is the content of $\S 2$; it allows us to replace the geometric arguments used in $[1,5]$ by a simpler linear algebra argument, which makes the invariant $c(G, X, L)$ easy to compute. We illustrate this in $\S 3$ and tabulate the value of $c(G, X, L)$ in many cases. For instance in the situation considered in $[1,5]$, our result says that if $V \subset \mathcal{A}_{g}$ is a subvariety of codimension at most $g$, then $S_{k}(V)$ is dense in $V$ for any $k$ between 1 and $g-1$. Another example of our result is that for every subvariety $V$ in $\mathcal{A}_{2^{9}}$ of codimension at most 10, the subset of points of

${ }^{*}$ Received March 20, 1998; accepted for publication April 16, 1998. This research is partially supported by grant DM95-02186 from the National Science Foundation.

$\dagger$ 4N32 David Rittenhouse Laboratory, University of Pennsylvania, Philadelphia, PA 19104, U.S.A. (chai@math.upenn.edu). 
$V$ corresponding to abelian varieties attached to a polarized $\mathrm{K} 3$ surface form a dense subset in $V$. The examples in $\S 3$ occupy more than half of this paper. The reader is advised to go over just one or two of them to see the results in action.

Although in most irreducible cases the invariant $c(G, X, L)$ is positive when $L$ is reasonably large, it is not always so; we give two such examples. In one example $G$ is of type $E_{6}$ and $L^{\text {ad }}$ is of type $D_{5}$. In another example $G$ is of type $E_{7}$, and $L^{\text {ad }}$ is of type $D_{6}$.

1. Formulation. We shall follow Deligne's point of view on Shimura varieties and think of a Shimura variety $M_{K}(G, X)$ as a parameter space of Hodge structures whose Hodge group (or, special Mumford-Tate group) is contained in $G$; see $[2,3$, 4]. The notation and convention in those papers will be generally followed. More specifically, let $G$ be a connected reductive linear algebraic group over $\mathbb{Q}$, let $X$ be a $G(\mathbb{R})$-conjugacy class of $\mathbb{R}$-homomorphisms from $\mathbb{S}=\operatorname{Res}_{\mathbb{C} / \mathbb{R}} \mathbb{G}_{m}$ to $G$ such that the following conditions are satisfied:

1. For one (hence for every) $h \in X$, under the adjoint representation $A d \circ h$ of $\mathbb{S}$ on $\mathfrak{g}=\operatorname{Lie}(G), \mathfrak{g}$ is a Hodge $\mathbb{Q}$-structure of type $\subseteq\{(1,-1),(0,0),(-1,1)\}$.

2. For one (hence for every) $h \in X, A d \circ h(\sqrt{-1})$ induces a Cartan involution on $G^{\text {ad }} \times$ Spec $\mathbb{Q} S p e c \mathbb{R}$.

3. Every nontrivial simple $\mathbb{Q}$-factor of $G$ is non-compact.

For every compact open subgroup $K \subset G\left(\mathbb{A}_{f}\right)=\prod_{p}^{\prime} G\left(\mathbb{Q}_{p}\right)$, the complex analytic variety $G(\mathbb{Q}) \backslash X \times G\left(\mathbb{A}_{f}\right) / K$ has a canonical structure of a complex quasi-projective variety; we denote it by $M_{K}(G, X)$. When $K$ is small enough (for instance if it is neat) $M_{K}(G, X)$ is smooth. Since the properties of $M_{K}(G, X)$ we consider do not depend on the choice of $K$, we shall assume for simplicity that $M_{K}(G, X)$ is smooth. Because the argument we use is entirely differential geometric, the canonical model and other arithmetic structures of $M_{K}(G, X)$ will not be used in this paper.

To every finite dimensional $\mathbb{Q}$-rational representation $\rho: G \rightarrow G L\left(V_{\rho}\right)$ of $G$ on a finite dimensional $\mathbb{Q}$-vector space $V_{\rho}$, there is a variation of $\mathbb{Q}$-rational Hodge structure over $X$ naturally attached to $\rho$; it descends to $M_{K}(G, X)$ if $K$ is small enough. For $G=G S p_{2 g}, X=\mathbb{H}_{g}^{ \pm}=$the Siegel upper-and-lower half space of genus g, $K \subset G S p_{2 g}(\hat{\mathbb{Z}})$ the principal congruence subgroup of level $\mathrm{n}$ and $\rho$ the standard representation, we get the family of Hodge structures $\underline{H}_{1}(A(\mathbb{C}), \mathbb{Q})$ over $\mathcal{A}_{g, n}$, where $f: A \rightarrow \mathcal{A}_{g, n}$ is the universal principally polarized abelian variety with level-nstructure over $\mathcal{A}_{g, n}, n \geq 3$.

The following construction is used in $[1,5]$. To every $\mathbb{C}$-vector subspace $W \subset$ $\operatorname{Lie}(A(\mathbb{C}))$ of dimension $k$, one associates to it its underlying $\mathbb{R}$-vector space, thought of as an $\mathbb{R}$-vector subspace of the $\mathbb{R}$-vector space $\underline{\mathrm{H}}_{1}(A(\mathbb{C}), \mathbb{R})$. This gives a map from the complex Grassmannian of $k$-dimensional subspaces of $\operatorname{Lie}(A(\mathbb{C}))$ to the real Grassmannian of $2 k$-dimensional subspaces of $\underline{\mathrm{H}}_{1}(A(\mathbb{C}), \mathbb{R})$. To adapt this construction to our setting, first observe that the only $2 k$-dimensional $\mathbb{R}$-subspaces of $\underline{\mathrm{H}}_{1}(A(\mathbb{C}), \mathbb{R})$ which appears are those such that the restriction of the polarization form to them are non-degenerate. So we can replace the target real Grassmanian space by the quotient $S p_{2 g}(\mathbb{R}) / S p_{2 k}(\mathbb{R}) \times S p_{2 g-2 k}(\mathbb{R})$. Notice that the source complex Grassmannian can be written as a quotient $U_{g}(\mathbb{R}) / U_{k}(\mathbb{R}) \times U_{g-k}(\mathbb{R})$, and that $U_{k}(\mathbb{R}) \times U_{g-k}(\mathbb{R})$ is the intersection of $S p_{2 g}(\mathbb{R}) / S p_{2 k}(\mathbb{R})$ with the maximal compact subgroup $U_{g}(\mathbb{R})$ of $S p_{2 g}(\mathbb{R})$. Hopefully the above discussion either motivates the definition and the construction below or illustrates them. 
Let $L$ be a $\mathbb{Q}$-rational reductive subgroups of $G$ such that there exists a homomorphism $h: \mathbb{S} \rightarrow G$ in $X$ which factorizes through $L$.

Definition 1.1. For an analytic subvariety $V \subseteq M_{K}(G, X), S(V, L)$ denotes the subset of $V$ consisting of all points $x \in V$ of the form $x=[(h, g)]$ with $h \in X$, $g \in G\left(\mathbb{A}_{f}\right)$ such that $h$ factorizes through $\gamma \cdot L \cdot \gamma^{-1}$ for some $\gamma \in G(\mathbb{Q})$.

REMARK. (i) Clearly $S(V, L)=S\left(V, L^{\prime}\right)$ if $L$ and $L^{\prime}$ are conjugate under $G(\mathbb{Q})$. The subset $S(V, L)$ is equal to the intersection of $S\left(M_{K}(G, X), L\right)$ with $V$.

(ii) The condition that $h$ factorizes through $A d(\gamma) \cdot L$ means that the special Mumford-Tate group for $h$ is contained in $A d(\gamma) \cdot L$. This justifies our assumption that $L$ is reductive, since the special Mumford-Tate group for any Hodge structure is reductive.

(iii) In the case $G=G S p_{2 g}, X=\mathbb{H}_{g}^{ \pm}$, and $L$ the intersection of $G$ with the product $G S p_{2 k} \times G S p_{2 g-2 k}$ diagonally embedded in $G L_{2 g}, S(V, L)$ is equal to the subset $S_{k}(V)$ of $V$ defined in the introduction.

We denote by $\mathfrak{C}$ the set of all $\mathbb{R}$-rational subgroups $L^{\prime}$ of $G$ conjugate to $L$ under $G(\mathbb{R})$; it has a natural structure as a real-analytic manifold. Let $\Pi$ denote the set consisting of all pairs $\left(h, L^{\prime}\right)$ with $h \in X, L^{\prime} \in \mathfrak{C}$ such that $h$ factorizes through $L^{\prime}$. Again $\Pi$ is a real-analytic manifold. The two natural projections $p r_{1}: \Pi \rightarrow X$ and $p r_{2}: \Pi \rightarrow \mathfrak{C}$ are both real-analytic maps. The group $G(\mathbb{R})$ operates on the left of $\Pi, X$ and $\mathfrak{C}$, and the two projections are both equivariant with respect to $G(\mathbb{R})$. For the given subvariety $V \subset M_{K}(G, X)$, let $\tilde{V}$ be the inverse image of $V$ in $\Pi$, and let $\phi: \tilde{V} \rightarrow \mathfrak{C}$ be the restriction of the projection $\Pi \rightarrow \mathfrak{C}$ to $\tilde{V}$.

Proposition 1. Assume that $\phi: \tilde{V} \rightarrow \mathfrak{C}$ is generically a submersion, i.e. $\phi$ is a submersion outside a nowhere-dense real-analytic subset $Z$ of $\tilde{V}$. Then $S(V, L)$ is dense in $V$ for the metric topology.

Proof. We only need to recall the well-known fact that $G(\mathbb{Q})$ is dense in $G(\mathbb{R})$ for the metric topology, and to observe that the projection map $\operatorname{pr}_{1}: \tilde{V} \rightarrow V$ is surjective. An elementary topological argument concludes the proof: For non-empty open subset $U$ of $V$, the image of $\operatorname{pr}_{1}^{-1}(U)-Z$ under $\phi$ is open in $\mathfrak{C}$, hence contains a $G(\mathbb{Q})$-conjugate of $L$.

Next we compute the image of the differential of the map $\phi: \tilde{V} \rightarrow \mathfrak{C}$. Let $y=\left(h, L_{1}\right)$ be a point of $\tilde{V}$ which lies over a smooth point of $V$. Then $y$ is a smooth point of $\tilde{V}$ as well. Denote by $N_{G}\left(L_{1}\right)$ the normalizer of $L_{1}$ in $G$, and let $N_{\mathfrak{g}}\left(L_{1}\right)=\operatorname{Lie}\left(N_{G}\left(L_{1}\right)\right)$ be its Lie algebra, which is also equal to the normalizer $N_{\mathfrak{g}}\left(\mathfrak{l}_{1}\right)$ of $\mathfrak{l}_{1}=\operatorname{Lie}\left(L_{1}\right)$ in $\mathfrak{g}=\operatorname{Lie}(G)$. We identify the tangent space of $\mathfrak{C}$ at the point $\left[L_{1}\right] \in \mathfrak{C}$ with $\mathfrak{g} / N_{\mathfrak{g}}\left(\mathfrak{l}_{1}\right)$. Let $\bar{V}$ be the inverse image of $V$ in $X$. The fiber at $h \in \bar{V}$ of $\tilde{V} \rightarrow \bar{V}$ maps under $\phi$ onto the set of all $K_{h}$-conjugates of $L_{1}$, where $K_{h}$ denotes the centralizer of $h$ in $G$. Therefore the differential of $\phi$ at the point $y=\left(h, L_{1}\right)$ sends the $p r_{1}$-vertical tangent space onto $\mathfrak{k}_{h}+N_{\mathfrak{g}}\left(\mathfrak{l}_{1}\right) / N_{\mathfrak{g}}\left(\mathfrak{l}_{1}\right)$, where $\mathfrak{k}_{h}=\operatorname{Lie}\left(K_{h}\right)$. Following the literature we denote by $\mathfrak{p}$ the $(-1)$-eigenspace of $A d(h(\sqrt{-1}))$ in $\mathfrak{g}$, identified with the tangents space of $X$ as usual. The tangent space of $\bar{V}$ at the point $h \in \bar{V}$ then become an $\mathbb{R}$-vector subspace of $\mathfrak{p}$, which we denote by $U$. The image of $d \phi$ at $\left(h, L_{1}\right)$ is equal to $\mathfrak{k}_{h}+U+N_{\mathfrak{g}}\left(\mathfrak{l}_{1}\right) / N_{\mathfrak{g}}\left(\mathfrak{l}_{1}\right)$ under our notation. The Lie algebra $\mathfrak{g}$ acquires a $\mathbb{R}$-Hodge structure under $A d \circ h$, and $\mathfrak{p}$ is a $\mathbb{R}$-Hodge substructure with Hodge decomposition $\mathfrak{p} \otimes_{\mathbb{R}} \mathbb{C}=\mathfrak{g}^{1,-1} \oplus \mathfrak{g}^{-1,1}$. Here $\mathfrak{g}^{1,-1}$ and $\mathfrak{g}^{-1,1}$ are the $(1,-1)$ and $(-1,1)$-components of $\mathfrak{g} \otimes_{\mathbb{R}} \mathbb{C}$ respectively, and $\mathfrak{g}^{-1,1}$ is identified with the complex tangent space of $X$ at $h$. Then $U \otimes_{\mathbb{R}} \mathbb{C}=U^{1,-1} \oplus U^{-1,1}$, with $U^{1,-1} \subseteq \mathfrak{g}^{1,-1}$ and $U^{-1,1} \subseteq \mathfrak{g}^{-1,1}$. Moreover $U^{-1,1}$ is the complex tangent space of $\bar{V}$ at the point $h$. Since $h$ factorizes through $L_{1} \subseteq N_{G}\left(L_{1}\right), N_{\mathfrak{g}}\left(\mathfrak{l}_{1}\right)$ is an $\mathbb{R}$-Hodge substructure of $\mathfrak{g}$; we 
write its Hodge decomposition as

$$
N_{\mathfrak{g}}\left(\mathfrak{l}_{1}\right) \otimes_{\mathbb{R}} \mathbb{C}=N_{\mathfrak{g}}\left(\mathfrak{l}_{1}\right)^{1,-1} \oplus N_{\mathfrak{g}}\left(\mathfrak{l}_{1}\right)^{0,0} \oplus N_{\mathfrak{g}}\left(\mathfrak{l}_{1}\right)^{-1,1} .
$$

Clearly $N_{\mathfrak{g}}\left(\mathfrak{l}_{1}\right)^{0,0}=N_{\mathfrak{g}}\left(\mathfrak{l}_{1}\right) \cap \mathfrak{k}_{h}$. Moreover $\mathfrak{k}_{h}+U+N_{\mathfrak{g}}\left(\mathfrak{l}_{1}\right)=\mathfrak{g}$ if and only if $U^{-1,1}+$ $N_{\mathfrak{g}}\left(\mathfrak{l}_{1}\right)^{-1,1}=\mathfrak{g}^{-1,1}$. We record the above in the following:

Proposition 2. Let $\left(h, L_{1}\right)$ be a point of $\tilde{V}$ lying over a smooth point of $V$. Identify the tangent space of $\bar{V}$ at $h$ with an $\mathbb{R}$-vector subspace $U \subseteq \mathfrak{p}_{h}=$ the (-1)eigenspace of $A d(h(\sqrt{-1}))$ in $\mathfrak{g} . \quad$ We identify the tangent space of $\mathfrak{C}$ at $L_{1}$ with $\mathfrak{g} / N_{\mathfrak{g}}\left(\mathfrak{l}_{1}\right)$. Then the image of the differential of $\phi: \tilde{V} \rightarrow \mathfrak{C}$ at $\left(h, L_{1}\right)$ is equal to $\mathfrak{k}_{h}+U+N_{\mathfrak{g}}\left(\mathfrak{l}_{1}\right)$, where $\mathfrak{k}_{h}$ denotes the $(+1)$-eigenspace of $A d(h(\sqrt{-1}))$ in $\mathfrak{g}$. Consequently $\phi$ is a submersion at $\left(h, L_{1}\right)$ if and only if $U^{-1,1}+N_{\mathfrak{g}}\left(\mathfrak{l}_{1}\right)^{-1,1}=\mathfrak{g}^{-1,1}$.

Corollary 1.1. Let $V, \tilde{V}, \bar{V}$ be as above. Suppose that there exists a point $h \in X$ lying over a smooth point of $V$ and an element $L_{1} \in \mathfrak{C}$ such that $h$ factorizes through $L_{1}$, and such that the tangent space $U^{-1,1}$ of the inverse image $\bar{V}$ of $V$ in $X$ at $h$ is transversal with $N_{\mathfrak{g}}\left(\mathfrak{l}_{1}\right)^{-1,1}$ in $\mathfrak{g}^{-1,1}$. Then $S(V, L)$ is dense in $V$.

REMARK. (i) We shall see that when the codimension of $V$ in $M_{K}(G, X)$ is smaller than or equal to a constant $c(G, X, L)$, then for every smooth point $[(h, g)]$ of $V$ with $h \in X, g \in G\left(\mathbb{A}_{f}\right)$, there exists an $L_{1} \in \mathfrak{C}$ satisfying the condition in the corollary.

(ii) Suppose that the condition of the corollary is not satisfied by any point $[(h, g)]$ in $V$. This gives us a system of equations satisfied by all points of $\bar{V}$. We can differentiate these equations to get linearized equations. In this paper we shall only use the vertical directions, although it is conceivable that differentiation in the horizontal directions may produce further information.

2. Further linearization. Let $G, X, L$ be as before. For an element $h: \mathbb{S} \rightarrow G$ in $X$, denote by $K_{h}$ the centralizer of $h$ in $G$. We abbreviate to $N$ the $(-1,1)$ component $N_{\mathfrak{g}}(\mathfrak{l})^{-1,1}$ of the normalizer $N_{\mathfrak{g}}(\mathfrak{l})$ of $\mathfrak{l}=\operatorname{Lie}(L)$ in $\mathfrak{g}$. Our discussion in the last section led us to make the following definition.

Definition 2.1. Let $L_{1}$ be a $G(\mathbb{R})$-conjugate of $L$ and $h: \mathbb{S} \rightarrow L_{1} \subseteq G$ be an element of $X$. When $N_{\mathfrak{g}}(\mathfrak{l})^{-1,1} \varsubsetneqq \mathfrak{g}^{-1,1}$, define $c\left(G, h, L_{1}\right)$ to be the largest nonnegative integer such that for every $\mathbb{C}$-vector subspace $U \subseteq \mathfrak{g}^{-1,1}$ of codimension $\leq c\left(G, h, L_{1}\right)$, there exists an element $k \in K_{h}(\mathbb{R})$ such that $\bar{U}+k \cdot N_{\mathfrak{g}}\left(\mathfrak{l}_{1}\right)^{-1,1} \cdot k^{-1}=$ $\mathfrak{g}^{-1,1}$. When $N_{\mathfrak{g}}(\mathfrak{l})^{-1,1}=\mathfrak{g}^{-1,1}, c\left(G, h, L_{1}\right)$ is defined to be $\infty$. Clearly $c\left(G, h, L_{1}\right)=$ $c\left(G, A d(g) \cdot h, A d(g) \cdot L_{1}\right)$ for every $g \in G(G(\mathbb{R})$. It is easy to check that in fact $c\left(G, h, L_{1}\right)=c\left(G, h^{\prime}, L_{2}\right)$ for any $G(\mathbb{R})$-conjugate $L_{2}$ of $L$ and any element $h^{\prime}: \mathbb{S} \rightarrow$ $L_{2} \subseteq G$ in $X$. So we write $c(G, X, \mathfrak{C})$ instead of $c\left(G, h, L_{1}\right)$.

Remark. (i) It is immediate from the Corollary of Proposition 2 that $S(V, L)$ is dense in $V$ for every analytic subvariety $V \subseteq M_{K}(G, X)$ of codimension at most $c(G, X, \mathfrak{C})$.

(ii) Clearly $c(G, h, L)$ can be computed after base change to $\mathbb{R}$, i.e. it is insensitive to the $\mathbb{Q}$-structure. It is also clear that the invariant $c\left(G, h, L_{1}\right)$ can be computed from the $K_{h}$-representation $\mathfrak{g}^{-1,1}$ and the $\mathbb{C}$-vector subspace $N_{\mathfrak{g}}\left(\mathfrak{l}_{1}\right)^{-1,1}$ in $\mathfrak{g}^{-1,1}$. This prompts us to make the following definition.

DEFINITION 2.2. Let $\rho$ be a real-analytic linear representation of a real-analytic Lie group $K$ on a finite dimensional $\mathbb{C}$-vector space $V_{\rho}$. Let $N$ be a $\mathbb{C}$-vector subspace of $V_{\rho}$. When $N \neq V_{\rho}$, define $d(K, \rho, N)$ to be the largest non-negative integer such that for every $\mathbb{C}$-vector subspace $W$ of $V_{\rho}$ of codimension at most $d(K, \rho, N)$, there exists an element $k \in K$ with $V_{\rho}=W+\rho(k) \cdot N$. When $N=V_{\rho}, d(K, \rho, N)$ is defined to be $\infty$. 
Remark. Clearly $c(G, h, L)=d\left(K_{h}, \mathfrak{g}^{-1,1}, N_{\mathfrak{g}}(\mathfrak{l})^{-1,1}\right)$ in the context of the two definitions above. Hence the results we have proved so far shows that the subset $S(V, L)$ of $V$ is dense in $V$ for every analytic subvariety $V \subseteq M_{K}(G, X)$ of codimension at most $d\left(K_{h}, \mathfrak{g}^{-1,1}, N_{\mathfrak{g}}(\mathfrak{l})^{-1,1}\right)$.

We list some trivial properties of the invariant $d(K, \rho, N)$ in the following lemma. The proofs are omitted.

Lemma 2.1. (1) $d(K, \rho, N)=d(K, \rho, \rho(k) \cdot N)$ for every $k \in K$.

(2) $d\left(K, \rho, N_{1}\right) \geq d\left(K, \rho, N_{2}\right)$ if $N_{1} \subseteq N_{2}$. $V_{\rho_{2}}$.

(3) $d\left(K, \rho_{1} \oplus \rho_{2}, N_{1} \oplus N_{2}\right) \leq \min \left(d\left(K, \rho_{1}, N_{1}\right), d\left(K, \rho_{2}, N_{2}\right)\right)$ if $N_{1} \subseteq V_{\rho_{1}}, N_{2} \subseteq$

(4) $d\left(K, \rho_{1} \oplus \rho_{2}, N_{1} \oplus V_{\rho_{2}}\right)=d\left(K, \rho_{1}, N_{1}\right)$.

(5) $d(K, \rho \otimes \lambda, N)=d(K, \rho, N)$ for every 1-dimensional representation $\lambda: K \rightarrow$ $\mathbb{C}^{\times}$of $K$. Here the representation space of $\rho \otimes \lambda$ is taken to be $V_{\rho}$, with $\rho \otimes \lambda(k) v=$ $\lambda(k) \rho(k) v$ for all $v \in V_{\rho}$.

LEMMA 2.2. Let $\rho_{1}, \rho_{2}$ be two complex linear representations of $K$ as above. Assume either that the real analytic Lie group $K$ is connected, or that $K$ is the group of real points of a connected real linear algebraic group $\tilde{K}$ and the two representations $\rho_{1}, \rho_{2}$ are both algebraic. Then

$$
d\left(K, \rho_{1} \oplus \rho_{2}, N_{1} \oplus N_{2}\right)=\min \left(d\left(K, \rho_{1}, N_{1}\right), d\left(K, \rho_{2}, N_{2}\right)\right)
$$

for any $\mathbb{C}$-vector subspace $N_{i} \subseteq V_{\rho_{i}}, i=1,2$.

Proof. We may assume that $N_{1} \neq V_{\rho_{1}}, N_{2} \neq V_{\rho_{2}}$. Let $W$ be a vector subspace of $V_{\rho_{1}} \oplus V_{\rho_{2}}$ of codimension at most $\min \left(d\left(K, \rho_{1}, N_{1}\right), d\left(K, \rho_{2}, N_{2}\right)\right)$. Consider the non-empty subsets $U_{i} \subset K$ consisting of all elements $k \in K$ such that $V_{\rho_{i}}=\rho_{i}(k)$. $N_{i}+\left(V_{\rho_{i}} \cap W\right), i=1,2$. Each $U_{i}$ is the complement of a closed real-analytic subset of $K$. So $U_{1} \cap U_{2} \neq \emptyset$ when $K$ a connect real analytic group. When $K$ is the real points of a connected real linear algebraic group $\tilde{K}$ and $\rho_{1}, \rho_{2}$ are algebraic, each $U_{i}$ is the set of real points of a non-empty Zariski open subset $\tilde{U}_{i}$ of $\tilde{K}$. So $\tilde{U}_{1} \cap \tilde{U}_{2} \neq \emptyset$, so $U_{1} \cap U_{2}=\left(\tilde{U}_{1} \cap \tilde{U}_{2}\right)(\mathbb{R}) \neq \emptyset$ since $\tilde{U}_{1} \cap \tilde{U}_{2}$ is unirational over $\mathbb{R}$. Clearly $W+\left(\rho_{1} \oplus \rho_{2}\right)(k) \cdot\left(N_{1} \oplus N_{2}\right)=V_{\rho_{1}} \oplus V_{\rho_{2}}$ for any $k \in U_{1} \cap U_{2}$ and the lemma is proved.

REMARK. In the situation we are interested in, $K$ is the centralizer of an element $h \in X$, therefore $K$ is a connected real linear algebraic group and Lemma 2.2 applies. This often reduces the computation of the invariant $c(G, X, \mathfrak{C})$ to the cases when $X$ is irreducible. Then Lemma 2.1 allows one to reduce further to the case when $G$ is simple over $\mathbb{R}$.

Proposition 3. Let $\rho$ be a finite dimension complex linear representation of a real Lie group $K$. Assume either that $K$ is a connected real-analytic group, or that $K$ $i s$ the group of real points of a connected real algebraic group $\tilde{K}$ and $\rho$ is an algebraic representation of $K$. Let $\mathfrak{k}=\operatorname{Lie}(K)$. Let $N, W$ be $\mathbb{C}$-vector subspaces of $V_{\rho}$. Then $\operatorname{dim}(W+N) \geq \operatorname{dim}(W+\rho(k) \cdot N)$ for all $k \in K$ if and only if $\rho(\mathfrak{k}) \cdot(N \cap W) \subseteq W+N$.

Proof. The proof is an exercise, but we give it here since it does not seem to be well-known. We first choose a basis $v_{1}, \ldots, v_{a}$ of $W \cap N$. Then extend it on the one hand to a basis $v_{1}, \ldots, v_{a}, w_{1}, \ldots, w_{b}$ of $W$, and on the other hand to a basis $v_{1}, \ldots, v_{a}, v_{a+1}, \ldots, v_{a+c}$ of $N$. For $1 \leq i \leq a$, consider the function $f_{i}: K \rightarrow$ $\bigwedge^{a+b+c+1}$ on $K$, defined by

$$
f_{i}(x)=\rho(x) v_{i} \wedge \rho(x) v_{a+1} \wedge \cdots \wedge \rho(x) v_{a+c} \wedge v_{1} \wedge \cdots \wedge v_{a} \wedge w_{1} \wedge \cdots \wedge w_{b}
$$


Clearly each $f_{i}$ is a real-analytic function on $K$, and it is a regular rational function on $K$ in the algebraic case. First suppose that $\operatorname{dim}(W+N) \geq \operatorname{dim}(W+\rho(k) \cdot N)$ for all $k \in K$. Then each $f_{i}(x)$ is identically 0 on $K$. Differentiating $f_{i}$, we see that $\rho(\mathfrak{k}) \cdot v_{i} \in W+N$ for each $i=1, \ldots, a$. Conversely, if $\rho(\mathfrak{k}) \cdot(N \cap W) \subseteq W+N$, then the derivatives of each $f_{i}$ are zero, hence $f_{i}$ is identically zero on $K$. The same argument shows that the function

$$
x \mapsto \rho(x) u_{1} \wedge \cdots \wedge \rho(x) u_{c+1} \wedge v_{1} \wedge \cdots \wedge v_{a} \wedge w_{1} \wedge \cdots \wedge w_{b}
$$

is identically zero on $K$ for any choice $u_{1}, \ldots, u_{c+1}$ of $c+1$ elements among the basis $v_{1}, \ldots, v_{a}, v_{a+1}, \ldots, v_{a+c}$ of $N$. This just means that $\operatorname{dim}(W+\rho(k) \cdot N) \leq a+b+c=$ $\operatorname{dim}(W+N)$ for all $k \in K$.

Corollary 2.1. Let $K, \rho$ be as in Proposition 3. Let $N$ be a vector subspace of $V_{\rho}$. Then

$$
d(K, \rho, N)=\min \left\{\operatorname{codim}\left(N_{1}, N\right) \mid N_{1} \subseteq N, N+\rho(\mathfrak{k}) \cdot N_{1} \varsubsetneqq V_{\rho}\right\} .
$$

REMARK. (i) This corollary turns the computation of $d(K, \rho, N)$ into a question in finite dimensional representations of Lie algebras, and is quite useful. Also notice that we can complexify the representation $\rho$ of the Lie algebra $\mathfrak{k}$ in computing $d(K, \rho, N)$.

(ii) Although it is not feasible to give the value of $d(K, \rho, N)$ in every case of interest, we will give many examples to illustrate our result. The irreducible representations of interest to us include the second symmetric product of the standard representation for $A_{n}$, the second exterior product of the standard representation of $A_{n}$, the tensor product of the two standard representations for $A_{m} \times A_{n}$, the standard representation of $B_{n}$, the standard representation of $D_{n}$, (one of the two of) the half-spin representation of $D_{5}$ and (one of the two of) the miniscule representation of $E_{6}$.

3. Examples. We asserted that the invariant

$$
c=c(G, X, \mathfrak{C})=c(G, h, L)=d\left(K_{h}, N_{\mathfrak{g}}(\mathfrak{l})^{-1,1}\right)
$$

is easy to compute in any given situation. In this section we illustrate this point in many examples. As we remarked before, this invariant depends only on the real groups underlying $G$, the $G(\mathbb{R})$-conjugacy class of the real group underlying $L$, and of course the space $X$. So from now on we assume that $G$ is a connected reductive linear algebraic group over $\mathbb{R}$, and that $L$ is a reductive subgroup of $G$ over $\mathbb{R}$. In all the examples below the connected components of $X$ are irreducible hermitian symmetric spaces, so $G$ is simple modulo center. Because of Lemma 2.1 (5), the value of $c$ does not change if we replace $G$ by $G^{\text {ad }}$, so we may freely change $G$ to $G^{\prime}$ with $G^{\text {ad }}=G^{\prime \text { ad }}$. It is well-known that $G$ is the inner twist by $A d(h(\sqrt{-1}))$ of its compact form, and $\mu_{h}=h \circ \mu$ is the fundamental coweight corresponding to a special vertex of the Dynkin diagram of the compact form of $G$, cf. [3] $\S 1$. In describing our examples, we shall give just a representative $G$ among those with the same derived group, indicate the conjugacy class of $\mu_{h}$, and give a description of (a representative of) $L$. We also give the Dynkin diagram for $(G, X)$; the vertex labeled with $\mu$ is the special vertex corresponding to the fundamental coweight $\mu_{h}$.

3.1. $\left(S p_{2 n}, S p_{2 n_{1}} \times \cdots \times S p_{2 n_{r}}\right), n_{1}+\cdots n_{r}=n$. First we bring up the case discussed in the introduction. Here 
- $G=G S p_{2 n}^{\mathrm{ad}}, X$ is the Siegel upper-and-lower half space of genus $n$. The homomorphism $h$ which sends an element $a+b \sqrt{-1} \in \mathbb{C}^{\times}$to

$$
\left(\begin{array}{ccccc}
a & -b & & & \\
b & a & & & \\
& & \ddots & & \\
& & & a & -b \\
& & & b & a
\end{array}\right)
$$

gives an element of $X$.

- The group $K_{h}$ is centrally isogenous to the unitary group $U(n)$.

- The Dynkin diagram is:

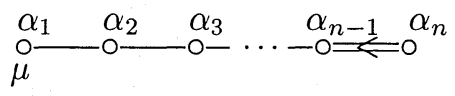

- The roots of $G$ are $\pm x_{i} \pm x_{j}, 1 \leq i \leq j \leq n$. The simple roots are

$$
\alpha_{1}=x_{1}-x_{2}, \ldots, \alpha_{n-1}=x_{n-1}-x_{n}, \alpha_{n}=2 x_{n} .
$$

- The subgroup $L$ in this example is the image of the diagonally embedded $S p_{2 n_{1}} \times \cdots \times S p_{2 n_{r}}$, where each $n_{i} \geq 1$, and $n_{1}+\cdots+n_{r}=n, r \geq 2$.

- The Lie algebra of the normalizer of $L$ in $G$ is equal to $l$ itself.

- After replacing $K_{h}$ by $U(n)$, the representation $\mathfrak{g}^{-1,1}$ becomes the second symmetric product of the standard representation of $U(n)$; the subspace $N \subset$ $\mathfrak{g}^{-1,1}$ is identified with

$$
S^{2}\left(\mathbb{C}^{n_{1}}\right) \oplus \cdots \oplus S^{2}\left(\mathbb{C}^{n_{r}}\right) \subset S^{2}\left(\mathbb{C}^{n}\right) .
$$

- The value of the invariant $c$ in this case is

$$
c=\min \left\{n_{i}+n_{j} \mid 1 \leq i \leq j \leq r\right\} .
$$

Our result says that for every analytic subvariety $V \subseteq \mathcal{A}_{n}$ of codimension at most this value of $c$, the set of all points in $V$ such that the corresponding principally polarized abelian variety is isogenous to the product of $r$ abelian varieties of dimension $n_{1}, \cdots, n_{r}$ is dense in $V$ for the metric topology.

Proof. We give a proof for the case $r=2$; the general case is similar. Moreover the same method applies to other examples, so we omit the proofs in most cases later. After complexifying the representation, we see that we are dealing with the second symmetric product of the standard representation $G L\left(V_{1} \oplus V_{2}\right)$, with $\operatorname{dim}\left(V_{1}\right)=n_{1}$, $\operatorname{dim}\left(V_{2}\right)=n_{2}, n=n_{1}+n_{2}$. The subspace $N$ is $S^{2}\left(V_{1}\right) \oplus S^{2}\left(V_{2}\right)$. We choose a basis $e_{1}, \ldots, e_{n_{1}}$ for $V_{1}$ and a basis $e_{n_{1}+1}, \ldots, e_{n_{1}+n_{2}}$ for $V_{2}$. This gives us a diagonal maximal torus in $G L\left(V_{1} \oplus V_{2}\right)$. The representation space $S^{2}\left(V_{1} \oplus V_{2}\right)$ has weights $x_{a}+x_{b}, 1 \leq a \leq b \leq n_{1}+n_{2}$, each with multiplicity one. The subspace $N$ is a direct sum of weight spaces, with weights $x_{a}+x_{b}$ with $1 \leq a \leq b \leq n_{1}$ and $x_{\alpha}+x_{\beta}$ with $n_{1}+1 \leq \alpha \leq \beta \leq n_{1}+n_{2}$.

First we show that $c \leq n_{1}+n_{2}$. Let $N_{1}$ be the direct sum of weight spaces with weights $x_{a}+x_{b}$ with $2 \leq a \leq b \leq n_{1}$ and $x_{\alpha}+x_{\beta}$ with $n_{1}+2 \leq \alpha \leq \beta \leq n_{1}+n_{2}$. Clearly $x_{1}+x_{n_{1}+1}$ is not a weight of $\mathfrak{g l}_{n} \cdot N_{1}+N$, so $\mathfrak{g l}_{n} \cdot N_{1}+N \varsubsetneqq S^{2}\left(V_{1} \oplus V_{2}\right)$. Hence $c \leq \operatorname{codim}\left(N_{1}, N\right)=n_{1}+n_{2}$.

Conversely, let $W \subseteq N$ be a subspace of codimension at most $n_{1}+n_{2}-1$. The intersection of $W$ with the direct sum of weight spaces with weights $x_{1}+x_{b}$ with 
$1 \leq b \leq n_{1}$ and $x_{n_{1}}+x_{\beta}$ with $n_{1}+1 \leq \beta \leq n_{2}$ contains a non-zero vector $q$. One quickly sees that $\mathfrak{g l}_{n} \cdot q+N$ contains the weight space with weight $x_{1}+x_{n_{1}+1}$. Similarly one sees that $\mathfrak{g l}_{n} \cdot W+N$ contains every weight space $x_{a}+x_{\alpha}$ with $1 \leq a \leq n_{1}$, $n_{1}+1 \leq \alpha \leq n_{1}+n_{2}$. Hence $\mathfrak{g l}_{n} \cdot W+N=S^{2}\left(V_{1} \oplus V_{2}\right)$.

3.2. $\left(S p_{2 n}, \mathrm{U}(p, q)\right), p+q=n$.

- In this example, the pair $(G, X)$ is essentially the same as in Example 3.1 above, except that we take $G$ to be $G S p_{2 n}$, rather than the adjoint group of it. So $K$ is isogenous to $\mathrm{U}(n) \times \mathrm{U}(1)$.

- The group $L$ is isogenous to $U(p, q) \times U(1)$ via a central isogeny, $p+q=n$. Its derived group $L^{\text {der }}$ has roots $\pm\left(x_{a}-x_{b}\right)$ with $1 \leq a<b \leq p$ and $\pm\left(x_{\alpha}-x_{\beta}\right)$ with $p+1 \leq \alpha<\beta \leq p+q$. We assume that $p \leq q$.

The symplectic embedding $L \hookrightarrow G$ arises in a PEL-type Shimura variety situation as follows. Start with an imaginary quadratic field $K$, a $2 n$-dimensional $\mathbb{Q}$-vector space $H$ with a $K$-action, a non-degenerate alternating pairing $\langle$, on $H$, and a $\mathbb{C}$-action on $H \otimes_{\mathbb{Q}} \mathbb{R}$. Assume that the involution on $\operatorname{End}(H)$ defined by the alternating form $\langle$,$\rangle leaves the image of K$ and $\mathbb{C}$ stable, and induces the complex conjugation on them. Moreover, assume that the bilinear form

$$
\left(v_{1}, v_{2}\right) \mapsto\left\langle v_{1},-i \cdot v_{2}\right\rangle
$$

on $H \otimes_{\mathbb{Q}} \mathbb{R}$ is symmetric and positive definite. The algebra $K \otimes_{\mathbb{Q}} \mathbb{C} \cong \mathbb{C} \times \mathbb{C}$ operates on $H \otimes_{\mathbb{Q}} \mathbb{R}$, and has $\mathbb{R}$-dimension $2 p, 2 q$ when localized at the two maximal ideals of $K \otimes \mathbb{Q} \mathbb{C} \cong \mathbb{C} \times \mathbb{C}$.

Such a PEL-data gives a simple algebra with involution $\left(\operatorname{End}_{K}(H), *\right)$, which gives rise to the symplectic embedding $L \subset G$. With the $\mathbb{C}$-action coming from the $\mathbb{C}^{\times}$-action as in Example 3.1, we may assume that the $K \otimes_{\mathbb{Q}} \mathbb{R} \cong \mathbb{C}$ action on $H \otimes_{\mathbb{Q}} \mathbb{R}$ is such that $a+b \sqrt{-1} \in \mathbb{C}$ operates via the matrix

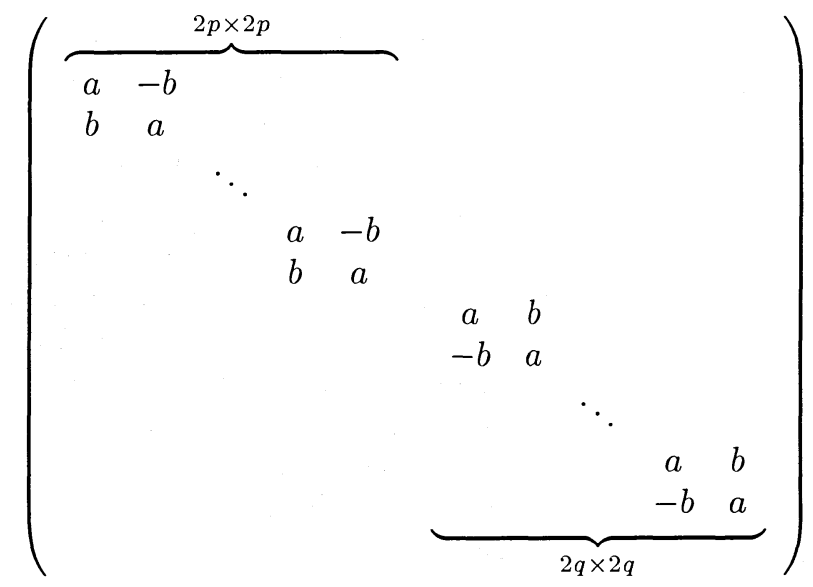

- The roots of $L$ are $\pm\left(x_{a}-x_{b}\right)$ with $1 \leq a<b \leq p, \pm\left(x_{\alpha}-x_{\beta}\right)$ with $p+1 \leq$ $\alpha<\beta \leq p+q$, and $\pm\left(x_{a}+x_{\beta}\right)$ with $1 \leq a \leq p$ and $p+1 \leq \beta \leq p+q$.

- The Lie algebra of the normalizer of $\mathfrak{l}=\operatorname{Lie}(L)$ is equal to $\mathfrak{l}$ itself. The subspace $N=\mathfrak{I}^{-1,1}$ has dimension $p q$, with roots $\left(x_{a}+x_{\beta}\right)$ with $1 \leq a \leq p$ and $p+1 \leq \beta \leq p+q$.

- $c=p=\min (p, n-p)$. 
The direct sum of the weight spaces in $N$ with weights $\left(x_{a}+x_{\beta}\right)$, where $2 \leq a \leq p$ and $p+1 \leq \beta \leq p+q$, is a subspace $N_{1}$ of codimension $p$ in $N$. Moreover the weight space for $2 x_{1}$ is not contained in $N_{1}+\mathfrak{g l}_{n} \cdot N_{1}$. This shows that $c \leq p$. The other half of the proof is omitted.

3.3. $(S O(2,2 n-1), S O(2,2 m-1) \times S O(2 n-2 m)), m<n$.

- In this example, $G$ is equal to $S O(2,2 n-1)$, and $L$ is the standardly embedded $S O(2,2 m-1) \times S O(2 n-2 m)$.

- The Dynkin diagram is

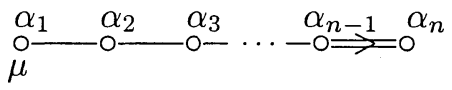

- The roots of $G$ are $\pm x_{i} \pm x_{j}$ with $1 \leq i<j \leq n$, and $x_{i}$ with $1 \leq i \leq n$. The simple roots are

$$
\alpha_{1}=x_{1}-x_{2}, \ldots \alpha_{n-1}=x_{n-1}-x_{n}, \alpha_{n}=x_{n} .
$$

The coweight $\mu_{h}$ corresponds to the only special vertex $\alpha_{1}$.

- The maximal compact subgroup $K$ is isomorphic to $S O(2) \times S O(2 n-1)$. The roots of $K^{\text {der }}$ are $\pm x_{i} \pm x_{j}$ with $2 \leq i<j \leq n$, and $x_{i}$ with $2 \leq i \leq n$. The representation $\mathfrak{g}^{-1,1}$ of $K$ has weights $x_{1}$ and $x_{1} \pm x_{a}$ with $2 \leq a \leq n$. As a representation of $K^{\text {der }} \cong S O(2 n-1)$ it is isomorphic to the standard representation.

- The roots of $L$ are $\pm x_{i} \pm x_{j}$ with $1 \leq i<j \leq m, \pm x_{i} \pm x_{j}$ with $m+1 \leq i<$ $j \leq n, x_{i}$ with $1 \leq i \leq m$. The normalizer of $\mathfrak{l}$ in $\mathfrak{k}$ is equal to $\mathfrak{l}$.

- The subspace $\mathfrak{l}^{-1,1}$ of $\mathfrak{g}^{-1,1}$ has weights $x_{1}$ and $x_{1} \pm x_{a}$ with $2 \leq a \leq m$.

- The invariant $c=2 m-2$.

3.4. $\left(S p_{2^{n}}, S O(2,2 n-1)\right)$. The roots and Dynkin diagram for $B_{n}$ are already recalled in Example 3.3 above. Here we start with a group $L$ of type $B_{n}$ and embed it in a symplectic group. An instance of this embedding is the Kuga-Satake construction of abelian varieties associated to a polarized K3 surface using the Clifford algebra.

- The group $L$ is the Spin double cover of $S O(2,2 n-1)$. The spin representation is the fundamental representation of $L$ with highest weight $\frac{1}{2}\left(x_{1}+\cdots+x_{n}\right)$. It has dimension $2^{n}$. Its weights are

$$
x_{\nu}=\frac{1}{2} \sum_{1 \leq \epsilon \leq n} \epsilon(i) x_{i},
$$

where $\epsilon=(\epsilon(1), \ldots, \epsilon(n))$, with each $\epsilon(i)= \pm 1$. Let $\Lambda$ be the abelian group defined by $2^{n}$ generators $\lambda_{\epsilon}$, where the $\epsilon$ 's are as above, and $2^{n-1}$ relations $\lambda_{\epsilon}+\lambda_{-\epsilon}=0$. Clearly $\Lambda$ is a free abelian group of rank $2^{n-1}$. This spin representation gives an embedding of $L$ into $S p_{2^{n}}$. Let the weights of the standard representation of $S p_{2^{n}}$ be the $2^{n}$ generators $\lambda_{\epsilon}$, and the spin representation is such that the weight $\lambda_{\epsilon}$ of $G$ goes to the weight $x_{\lambda}$ of $L$. The roots of $G$ are of course the $\lambda_{\epsilon_{1}}-\lambda_{\epsilon_{2}}$ 's.

- The coweight $\mu_{h}$ of $L$ corresponds to the homomorphism which sends $x_{\epsilon}$ to $\frac{1}{2} \epsilon(1)$, hence also the the homomorphism which sends $\lambda_{\epsilon}$ to $\frac{1}{2} \epsilon(1)$. From this one see that $\mathrm{g}^{-1,1}$ has weights $\lambda_{\epsilon_{1}}-\lambda_{\epsilon_{2}}$ with $\epsilon_{1}(1)=1$ and $\epsilon_{2}(1)=-1$.

- We have $\operatorname{Lie}\left(N_{G}(L)\right)=\operatorname{Lie}(L)$ since the spin representation is irreducible. The embedding $N=\mathfrak{l}^{-1,1} \hookrightarrow \mathfrak{g}^{-1,1}$ is determined from the map between the weights by duality. 
- We have $c=n$ in the present situation.

Let $N_{1} \subset N$ be the sum of weight spaces with weights $x_{1}+x_{2}, \ldots x_{1}+x_{n}$. Then $N_{1}$ has codimension $n$ in $N$, and one checks that $N+\mathfrak{k} \cdot N_{1} \subsetneq \mathfrak{g}^{-1,1}$. Hence $c \leq n$. The other part of the proof is omitted.

3.5. $\left(\mathrm{U}(p, q), \mathrm{U}\left(p_{1}, q_{1}\right) \times \cdots \mathrm{U}\left(p_{r}, q_{r}\right)\right), \sum p_{i}=p, \sum q_{i}=q$.

- In this example the real group $G$ is equal to $\mathrm{U}(p, q)$, defined by the diagonal hermitian form with signature $(p, q)$. The subgroup $L$ is equal to $\mathrm{U}\left(p_{1}, q_{1}\right) \times$ $\left.\cdots \mathrm{U}\left(p_{r}, q_{r}\right)\right)$, with $p_{1}+\cdots+p_{r}=p, q_{1}+\cdots+q_{r}=q$, diagonally embedded in $G$.

- The Dynkin diagram for $(G, X)$ is

$$
\underset{\circ}{\alpha_{1}} \stackrel{\alpha_{2}}{\circ} \ldots-{ }_{\mu}^{\alpha} \underline{\alpha_{p}} \ldots \stackrel{\alpha_{n-1}}{\alpha_{0}} \alpha_{p+q-1}
$$

- The simple roots of $G$ are $\alpha_{1}=x_{1}-x_{2}, \ldots, \alpha_{p+q-1}=x_{p+q-1}-x_{p+q}$. Similarly for each $\left.\mathrm{U}_{(} p_{i}, q_{i}\right)$. The maximal compact subgroup $K$ of $G$ is $\mathrm{U}(p) \times \mathrm{U}(q)$. The normalizer of the $\mathfrak{l}$ in $\mathfrak{g}$ is equal to $\mathfrak{l}$.

- The weights of $\mathfrak{g}^{-1,1}$ are $x_{a}-x_{\beta}$, with $1 \leq a \leq p$ and $p_{1} \leq \beta \leq p+q$. As a representation of $K$ it is isomorphic to the standard representation of $\mathrm{U}(p)$ tensored with the dual of the standard representation of $\mathrm{U}(q)$. The weights of $\mathfrak{l}^{-1,1}$ consists of those $x_{a}-x_{\beta}$ such that there exists an $i$ between 1 and $r$, with $p_{1}+\cdots+p_{i-1}+1 \leq a \leq p_{1}+\cdots+p_{i}$ and $q_{1}+\cdots+q_{i-1}+1 \leq \beta \leq q_{1}+\cdots+q_{i}$.

- The invariant $c=\min \left\{\min \left(p_{i}, q_{i}\right) \mid p_{i} \cdot q_{i} \neq 0,1 \leq i \leq r\right\}$.

3.6. $(S O(2,2 n-2), S O(2,2 m-2) \times S O(2 n-2 m)), m<n$.

- The Dynkin diagram for $(G, X)$ is

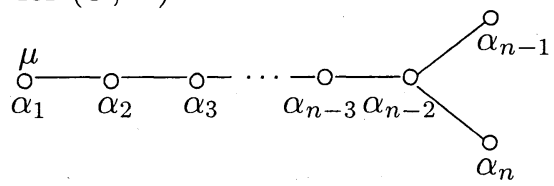

- The real group $G$ is equal to $S O(2,2 n-2)$, defined by the diagonal quadratic form with signature $(2,2 n-2)$. The reductive subgroup $L$ is equal to $S O(2$, $2 m-2) \times S O(2 n-2 m), m<n$, diagonally embedded in $G$.

- The roots of $G$ are $\pm x_{i} \pm x_{j}, 1 \leq i<j \leq n$. The simple roots are $\alpha_{1}=$ $x_{1}-x_{2}, \ldots, \alpha_{n-1}=x_{n-1}-x_{n}, \alpha_{n}=x_{n-1}+x_{n}$.

- The maximal compact subgroup $K$ of $G$ is $S O(2) \times S O(2 n-2)$. The compact roots are $\pm x_{i} \pm x_{j}, 2 \leq i<j \leq n$. The normalizer of $\mathfrak{l}$ in $\mathfrak{g}$ is equal to $\mathfrak{l}$.

- The weights of $\mathfrak{g}^{-1,1}$ are $x_{1} \pm x_{2}, \ldots, x_{1} \pm x_{n}$. As a representation of $S O(2 n-$ $2)=K^{\text {der }}$ it is isomorphic to the standard representation of $S O(2 n-2)$. The subspace $N=\mathfrak{l}^{-1,1}$ has weights $x_{1} \pm x_{2}, \ldots, x_{1} \pm x_{m}$.

- The invariant $c=2 m-3$

3.7. $\left(D_{n}^{\mathbb{H}}, D_{n_{1}}^{\mathbb{H}} \times \cdots D_{n_{r}}^{\mathbb{H}}\right), n_{1}+\cdots+n_{r}=n$.

- The Dynkin diagram for $(G, X)$ is

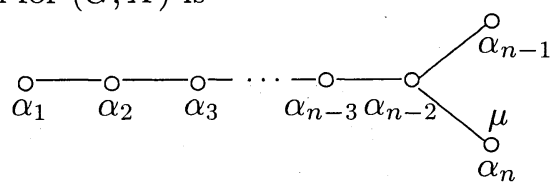

- This hermitian symmetric space is denoted $D_{n}^{\mathbb{H}}$ in [3]. It occurs for PEL-typeD Shimura varieties. The subgroup $L$ considered here is a diagonally embedded reductive subgroup, which is a product of groups of type $D_{n_{1}}^{\mathbb{H}}, \ldots D_{n_{r}}^{\mathbb{H}}$, 
where $r \geq 2$ and each $n_{i} \geq 1$.

- The roots and simple roots of $G$ are the same as in Example 3.6 above. The maximal compact subgroup $K$ of $G$ is isogenous to $\mathrm{U}(n)$ via a central isogeny. The compact roots are $\pm\left(x_{i}-x_{j}\right), 1 \leq i<j \leq n$. The roots of $L$ consists of those $\pm x_{a} \pm x_{b}$ such that there exists an $i, 1 \leq i \leq r$, with $n_{1}+\cdots+n_{i-1}+1 \leq a<b \leq n_{1}+\cdots+n_{i}$.

- The weights for $\mathfrak{g}^{-1,1}$ are $x_{i}+x_{j}, 1 \leq i<j \leq n$. Each weight space has dimension one. As a $\mathfrak{k}$-representation it is isomorphic to the second exterior product of the standard representation of $\mathfrak{u}(n)$.

- The normalizer of $\mathfrak{l}$ in $\mathfrak{g}$ is equal to $\mathfrak{l}$. The subspace $\mathfrak{l}^{-1,1}$ has weights $x_{a}+x_{b}$, where there exists an $i, 1 \leq i \leq r$, with $n_{1}+\cdots+n_{i-1}+1 \leq a<b \leq$ $n_{1}+\cdots+n_{i}$.

- The invariant $c=\min \left\{n_{i}+n_{j}-2 \mid i \neq j\right\}$.

3.8. $\left(E_{6}, D_{5}^{\mathbb{R}}\right)$. Something will be glaringly missing if we do not include exceptional groups in our examples. For hermitian symmetric domains, the only exceptional groups that appears are $E_{6}$ and $E_{7}$. We first discuss the $E_{6}$ case.

- The Dynkin diagram is

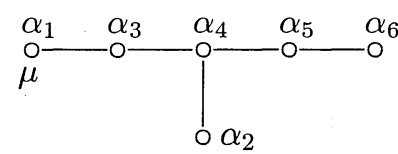

- $G$ is adjoint of type $E_{6}$. The root space is

$$
\left\{\sum_{1 \leq i \leq 8} \xi_{i} \cdot x_{i} \mid \xi_{6}=\xi_{7}=-\xi_{8}\right\}
$$

with inner product induced by the standard Euclidean inner product. The roots are

$$
\pm x_{i} \pm x_{j} \quad 1 \leq i<j \leq 5
$$

and

$$
\pm \frac{1}{2}\left(x_{8}-x_{7}-x_{6}+\sum_{1 \leq i \leq 5}(-1)^{\nu(i)} x_{i}\right)
$$

with $\sum_{1 \leq i \leq 5} \nu(i) \equiv 0(\bmod 2)$. The simple roots are

$$
\begin{aligned}
& \alpha_{1}=\frac{1}{2}\left(x_{1}-x_{2}-x_{3}-x_{4}-x_{5}-x_{6}-x_{7}+x_{8}\right) \\
& \alpha_{2}=x_{1}+x_{2} \\
& \alpha_{3}=x_{2}-x_{1} \\
& \alpha_{4}=x_{3}-x_{2} \\
& \alpha_{5}=x_{4}-x_{3} \\
& \alpha_{6}=x_{5}-x_{4}
\end{aligned}
$$

- There are two special vertices, $\alpha_{1}$ and $\alpha_{6}$, exchanged under an outer automorphism. Here the coweight $\mu_{h}$ corresponds to the special vertex $\alpha_{1}$.

- The maximal compact subgroup $K$ is isogenous to $S O(10) \times S O(2)$ via a central isogeny. The compact roots are $\pm x_{i} \pm x_{j}$ with $1 \leq i<j \leq 5$. 
- $\mathfrak{g}^{-1,1}$ has weights

$$
\frac{1}{2}\left(x_{8}-x_{7}-x_{6}+\sum_{1 \leq i \leq 5}(-1)^{\nu(i)} x_{i}\right)
$$

with $\sum_{1 \leq i \leq 5} \nu(i) \equiv 0(\bmod 2)$. This representation of $\left.\operatorname{Lie}\left(K^{\mathrm{ad}}\right)\right) \cong \mathfrak{s o}(10)$ is the half-spin representation of $\mathfrak{s o}(10)$ of dimension 16 . Each weight space is one-dimensional.

- In this example, the subgroup $L \subset G$ is of type $D_{5}$, with simple roots $\alpha_{1}, \alpha_{2}$, $\alpha_{3}, \alpha_{4}, \alpha_{5}$. The roots of $L$ are $\pm x_{i} \pm x_{j}, 1 \leq i<j \leq 4$ and

$$
\pm \frac{1}{2}\left(x_{8}-x_{7}-x_{6}-x_{5}+\sum_{1 \leq i \leq 4}(-1)^{\nu(i)} x_{i}\right)
$$

with $\sum_{1 \leq i \leq 4} \nu(i) \equiv 1(\bmod 2)$. The image of the homomorphism $h$ factorizes through $L$, giving rise to a hermitian symmetric space of type $D_{5}^{\mathbb{R}}$.

- The normalizer $N_{\mathfrak{g}}(\mathfrak{l})$ of $\mathfrak{l}=\operatorname{Lie}(L)$ in $\mathfrak{g}$ is equal to $\mathfrak{l}$ itself.

- The subspace $N_{\mathfrak{g}}(\mathfrak{l})^{-1,1}$ of $\mathfrak{g}^{-1,1}$ has dimension 8; its weights are

$$
\frac{1}{2}\left(x_{8}-x_{7}-x_{6}-x_{5}+\sum_{1 \leq i \leq 4}(-1)^{\nu(i)} x_{i}\right)
$$

with $\sum_{1<i<4} \nu(i) \equiv 1(\bmod 2)$.

- Regarded as a representation of the Lie algebra of the derived group of $K$, the weights of $\mathfrak{g}^{-1,1}$ are $\frac{1}{2}\left(\sum_{1 \leq i \leq 5}(-1)^{\nu(i)} x_{i}\right)$, with $\sum_{1 \leq i \leq 5} \nu(i) \equiv 0(\bmod 2)$. The weights of the subspace $N=N_{\mathfrak{g}}(\mathfrak{l})^{-1,1}$ are those for which $\nu(5)$ is odd.

- $c=4$ in this example.

Proof. The direct sum $N_{1}$ of the weight spaces with weights $\frac{1}{2}\left(x_{1}+x_{2}+x_{3}-\right.$ $\left.x_{4}-x_{5}\right), \frac{1}{2}\left(x_{1}+x_{2}-x_{3}+x_{4}-x_{5}\right), \frac{1}{2}\left(x_{1}-x_{2}+x_{3}+x_{4}-x_{5}\right), \frac{1}{2}\left(-x_{1}+x_{2}+\right.$ $\left.x_{3}+x_{4}-x_{5}\right)$ has codimension 4 in $\mathfrak{g}^{-1,1}$, and $N+\mathfrak{k} \cdot N_{1}$ does not contain the weight space of $\frac{1}{2}\left(-x_{1}-x_{2}-x_{3}-x_{4}+x_{5}\right)$. So $c \leq 4$. Conversely let $W$ be a subspace of $N$ of codimension at most 4 . Then the intersection of $W$ with the direct sum of weight spaces with weights $\frac{1}{2}\left(-x_{1}-x_{2}-x_{3}+x_{4}-x_{5}\right)$, $\frac{1}{2}\left(-x_{1}-x_{2}+x_{3}-x_{4}-x_{5}\right), \frac{1}{2}\left(-x_{1}+x_{2}-x_{3}-x_{4}-x_{5}\right), \frac{1}{2}\left(x_{1}-x_{2}-x_{3}-x_{4}-x_{5}\right)$ is not zero. So $\mathfrak{k} \cdot W$ contains the weight space of $\frac{1}{2}\left(-x_{1}-x_{2}-x_{3}-x_{4}+x_{5}\right)$. Similarly $\mathfrak{k} \cdot W$ contains the weight space of the other three weights with $\nu(5)$ even.

3.9. $\left(E_{6}, A_{5}\right)$. In this example, the pair $(G, X)$ is the same as in example 3.8 above; the subgroup $L$ is different.

- The subgroup $L \subset G$ is isogenous to $\mathrm{U}(1,5)$, so $L^{\text {ad }}$ is of type $A_{5}$. The simple roots of $L$ are $\alpha_{1}, \alpha_{3}, \alpha_{4}, \alpha_{5}, \alpha_{6}$. There are 20 compact roots among them; they are $\pm\left(x_{i}-x_{j}\right)$ with $1 \leq i<j \leq 5$. The normalizer of $\mathfrak{l}$ in $\mathfrak{g}$ is equal to $\mathfrak{l}$ itself.

- The subspace $N=N_{\mathfrak{g}}(\mathfrak{l})^{-1,1}=\mathfrak{l}^{-1,1}$ of $\mathfrak{g}^{-1,1}$ is 5 -dimensional, with weights $\frac{1}{2}\left(x_{1}-x_{2}-x_{3}-x_{4}-x_{5}\right), \frac{1}{2}\left(-x_{1}+x_{2}-x_{3}-x_{4}-x_{5}\right), \frac{1}{2}\left(-x_{1}-x_{2}+x_{3}-x_{4}-x_{5}\right)$, $\frac{1}{2}\left(-x_{1}-x_{2}-x_{3}+x_{4}-x_{5}\right), \frac{1}{2}\left(-x_{1}-x_{2}-x_{3}-x_{4}+x_{5}\right)$. One sees immediately that the the weight space for $\frac{1}{2}\left(x_{1}+x_{2}+x_{3}+x_{4}+x_{5}\right)$ is not contained in $\mathfrak{k} \cdot N$. Hence

- $c=0$. 
3.10. $\left(E_{7}, D_{6}^{\mathbb{R}}\right)$. The other exceptional group to deal with is $E_{7}$.

- The Dynkin diagram is

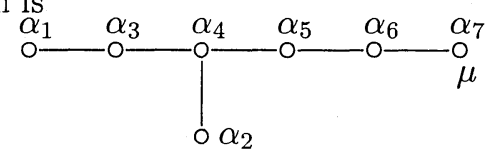

- $G$ is adjoint of type $E_{7}$. The root space is

$$
\left\{\sum_{1 \leq i \leq 8} \xi_{i} \cdot x_{i} \mid \xi_{7}+\xi_{8}=0\right\}
$$

with inner product induced by the standard Euclidean inner product. The roots are

$$
\pm x_{i} \pm x_{j} \quad 1 \leq i<j \leq 6 ; \quad \pm\left(x_{7}-x_{8}\right)
$$

and

$$
\pm \frac{1}{2}\left(x_{8}-x_{7}-x_{6}+\sum_{1 \leq i \leq 6}(-1)^{\nu(i)} x_{i}\right)
$$

with $\sum_{1 \leq i \leq 6} \nu(i) \equiv 2(\bmod 2)$. The simple roots are

$$
\begin{aligned}
& \alpha_{1}=\frac{1}{2}\left(x_{1}-x_{2}-x_{3}-x_{4}-x_{5}-x_{6}-x_{7}+x_{8}\right) \\
& \alpha_{2}=x_{1}+x_{2} \\
& \alpha_{3}=x_{2}-x_{1} \\
& \alpha_{4}=x_{3}-x_{2} \\
& \alpha_{5}=x_{4}-x_{3} \\
& \alpha_{6}=x_{5}-x_{4}
\end{aligned}
$$

The highest root is

$$
\tilde{\alpha}=x_{8}-x_{7}=2 \alpha_{1}+2 \alpha_{2}+3 \alpha_{3}+4 \alpha_{4}+3 \alpha_{5}+2 \alpha_{6}+\alpha_{7} .
$$

- The coweight $\mu_{h}$ corresponds to the only special vertex $\alpha_{7}$. The compact subgroup $K^{\text {ad }}$ is of type $E_{6}$. It has 72 roots. They are

$$
\frac{1}{2}\left(x_{8}-x_{7}-x_{6}+\sum_{1 \leq i \leq 5}(-1)^{\nu(i)} x_{i}\right)
$$

with $\sum_{1 \leq i \leq 5} \nu(i) \equiv 0(\bmod 2)$, and $\pm x_{i} \pm x_{j}$ with $1 \leq i<j \leq 5$.

- The subgroup $L \subset G$ is a reductive subgroup of the same rank as $G$. Its adjoint group $L^{\text {ad }}$ is of type $D_{6}$, with simple roots $\alpha_{2}, \alpha_{3}, \ldots, \alpha 7$. The image of $h$ is contained in $L$, giving rise to a hermitian symmetric space of type $D_{6}^{\mathbb{R}}$. We have $N_{\mathfrak{g}}(\mathfrak{l})=\mathfrak{l}$. The roots of $\mathfrak{l}$ are $\pm x_{i} \pm x_{j}, 1 \leq i<j \leq 6$; there are 60 such roots.

- The representation $\mathfrak{g}^{-1,1}$ of $K$ is irreducible of dimension 27 . Its weights are $x_{8}-x_{7}, x_{6} \pm x_{i}$ with $1 \leq i \leq 5$ and

$$
\frac{1}{2}\left(x_{8}-x_{7}+x_{6}+\sum_{1 \leq i \leq 5}(-1)^{\nu(i)} x_{i}\right)
$$


with $\sum_{1 \leq i \leq 5} \nu(i) \equiv 1(\bmod 2)$; each weight space dimension one. As a representation of the Lie algebra of the derived group of $K$, it is the fundamental representation of $\operatorname{Lie}\left(K^{\text {der }}\right)$ corresponding to the vertex $\alpha_{1}$, a miniscule representation for $E_{6}$.

- The subspace $N=N_{\mathfrak{g}}(\mathfrak{l})^{-1,1}$ of $\mathfrak{g}^{-1,1}$ has dimension 10 . Its weights are $x_{6} \pm x_{i}, 1 \leq i \leq 5$. We have

$$
\mathfrak{k} \cdot N+N \varsubsetneqq \mathfrak{g}^{-1,1}
$$

since the sum does not contain the weight space for $x_{8}-x_{7}$. Hence - $c=0$.

\section{REFERENCES}

[1] E. Colombo And G. P. Pirola, Some density results for curves with non-simple jacobians, Math. Ann., 288:161-178, 1990.

[2] P. Deligne, Travaux de Shimura, In Séminaire Bourbaki 1970/71, volume 244 of Lecture Notes in Math., 1971. Exposé 389.

[3] P. Deligne, Variétés de Shimura: Interprétation modulair, et techniques de construction de modèles canoniques, In A. Borel and W. Casselman, editors, Automorphic Forms, Representations, and L-functions, Part 2, volume 33 of Proc. Symp. Pure Math., pages 247-290, Providence, R.I., 1979. Amer. Math. Soc.

[4] P. Deligne, Hodge cycles on abelian varieties, In Hodge Cycles, Motives, and Shimura Varieties, volume 900 of Lecture Notes in Math., pages 9-100. Springer-Verlag, 1982. Notes by J. S. Milne.

[5] E. IZADI, Density and completeness of subvarieties of moduli spaces of curves or abelian varieties, to appear in Math. Ann. 